\title{
EXPANDING SHELLS OF \\ NEUTRAL HYDROGEN AS BIRTHPLACES OF \\ STELLAR ASSOCIATIONS
}

\author{
R. SANCISI \\ Kapteyn Astronomical Institute, University of Groningen, \\ Groningen, The Netherlands
}

\begin{abstract}
Expanding shells of neutral hydrogen have been found connected with the associations Per OB2 and Sco OB2. The shells also contain dust and molecules. A model of the spatial configuration and the kinematics of gas, dust and stars is discussed. The stars of the associations lie outside, close to the shells; gas and stars move outward from a common centre of expansion. If the shells are interpreted as old supernova remnants which have been strongly decelerated by the interstellar medium, ages of the order of $1 \times 10^{6} \mathrm{yr}$ are obtained. A genetic relationship between stars and shells is suggested. The stars formed in the densest gas shell; afterwards they separated from the gas, as the shell was further slowed down by intervening interstellar matter. Limits are set on the time scale of star formation. The geometry and the motion of the shell at the moment of star formation account also for the kinematics of Per OB2 and Sco OB2.
\end{abstract}

\section{Introduction}

The study of neutral hydrogen in regions of young stellar associations provides information on the physical conditions of interstellar matter which favour star formation, and on the interaction between young stars and interstellar gas. Previous $21-\mathrm{cm}$ line studies (cf. Raimond, 1966) have established the existence of massive and dense concentrations of neutral hydrogen connected with some OB associations; however, they have failed to reveal the nature of the connection between stars and gas. In particular, no conclusive evidence has ever been found for expanding motions of the gas in the regions of the associations.

In this paper we report the results of a new, detailed study of neutral hydrogen in the regions of the associations Per OB2 and Sco OB2. These objects are known to be very young and in rapid expansion (Blaauw, 1964). Some of their characteristics are listed in Table I. Both lie in the solar neighbourhood, at intermediate galactic latitudes; their membership, state of motion and age are fairly well known. For all these reasons they represent ideal candidates for a detailed investigation of the interstellar neutral hydrogen in regions of recent star formation.

The 21-cm observations were made with the $25-\mathrm{m}$ radiotelescope at Dwingeloo (beamwidth 0.6 ; bandwidth $2 \mathrm{~km} \mathrm{~s}^{-1}$ ). The linear resolution is $3.5 \mathrm{pc}$ at the distance of Per OB2 and $1.8 \mathrm{pc}$ at that of Sco OB2. For this study the observational data have been displayed in three different sets of contour maps, which show the distribution of brightness temperature in longitude and velocity at a constant galactic latitude, in latitude and velocity at a constant longitude, and in longitude and latitude at a constant radial velocity.

In the following section we show that expanding shells of neutral hydrogen do exist 
TABLE I

General properties of the associations Per OB2 and Sco OB2

\begin{tabular}{|c|c|c|}
\hline Property & Per OB2 & Sco OB2 \\
\hline Position $l$ & $156^{\circ}$ to $165^{\circ}$ & $341^{\circ}$ to $2^{\prime}$ \\
\hline$b$ & $-11^{\circ}$ to $-21^{\prime}$ & $+10^{\circ}$ to +30 \\
\hline$\alpha(1950)$ & $3^{\mathrm{h}} 25^{\mathrm{m}}$ to $4^{\mathrm{h}} 4^{\mathrm{m}}$ & $15^{\mathrm{h}} 30^{\mathrm{m}}$ to $16^{\mathrm{h}} 35^{\mathrm{m}}$ \\
\hline$\delta(1950)$ & $+30^{\circ}$ to $+36^{\circ}$ & $-30^{\circ}$ to $-15^{\circ}$ \\
\hline Distance from the Sun $(\mathrm{pc})$ & 330 & 170 \\
\hline Distance from galactic plane $(\mathrm{pc})$ & -85 & +55 \\
\hline Mean radial velocity $(\mathrm{LSR})\left(\mathrm{km} \mathrm{s}^{-1}\right)$ & +15 & +6 \\
\hline Total mass in stars $\left(10^{3} M_{\odot}\right)$ & 1.5 & 1.9 \\
\hline Estimated average (from $\mathrm{cm}$ diagrams) & 4 & 10 \\
\hline age $\left(10^{6} \mathrm{yr}\right) \quad$ (from kinematic data) & 1.5 & 20 \\
\hline
\end{tabular}

Notes to Table I

All the data in the table are quoted from Blaauw $(1960,1964)$ with the exception of the mean radial velocities; these have been derived in this study from the available, best determined radial velocities of the individual stars.

Slightly lower values for the age of Per OB2 have been obtained recently by Lesh (1969) and by Maeder (1972). The age of Sco OB2 is probably much smaller than in the table; Maeder finds $4.5 \times 10^{6} \mathrm{yr}$.

in the regions of the associations Per OB2 and Sco OB2; from the optical and $\mathrm{H}_{\mathrm{I}}$ data we derive a model of the spatial and kinematical arrangement of stars and gas. In the last section we discuss a possible interpretation of the observed $\mathrm{H}$ I shells as old supernova remnants and derive some conclusions on the process and the time scale of star formation.

\section{Results of the Observations}

A few representative maps showing the observational data are reproduced in Figures 1 and 2. All radial velocities in this paper are with respect to the local standard of rest (LSR). A detailed discussion of the observations will be given elsewhere; we here restrict ourselves to a discussion of the main results.

\section{(a) EXPANDING SHELLS OF NEUTRAL HYDROGEN}

Large concentrations of neutral hydrogen, associated with complexes of dark nebulosity, exist in the regions of the associations Per OB2 and Sco OB2. They stand out for their small velocity dispersion and their large intensities. Their velocity and spatial structure indicate that they are shells forming part of expanding systems of gas.

Most of the hydrogen forming the expanding system is concentrated in a dense shell of roughly semispherical shape. The shells are visible in Figure 1. Figure 1a shows the distribution of brightness temperature, $T_{\mathrm{b}}$, in the longitude-velocity plane at constant galactic latitude, $b=-18^{\circ}$, through the association Per OB2. The semispherical shell is the high-intensity feature around longitude $l=160^{\circ}$, with the peak- 


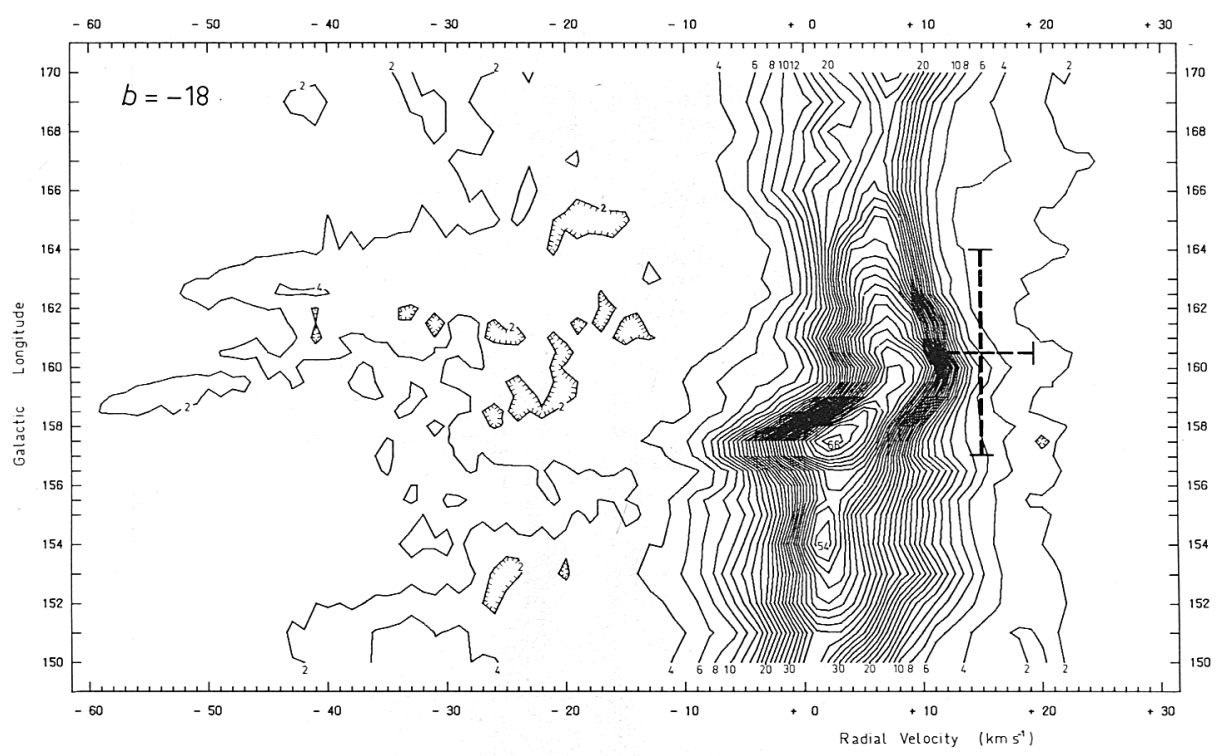

Fig. 1a. Map of contours of equal brightness temperature in a constant latitude cut $\left(b=-18^{\circ}\right)$ through the region of the association Per OB2. The contour interval is $2 \mathrm{~K}$. The high-intensity feature around longitude $l=160^{\circ}$, with the peak velocity gradient from 0 to $+8 \mathrm{~km} \mathrm{~s}^{-1}$, forms the semispherical shell discussed in the text and sketched in Figure 3. The vertical, broken line shows the longitude extent of the association at the mean radial velocity $\left(+15 \mathrm{~km} \mathrm{~s}^{-1}\right.$, LSR $)$; the horizontal line gives the average velocity residual.

velocity gradient from 0 to $+8 \mathrm{~km} \mathrm{~s}^{-1}$. The velocity field of this feature has been already described in detail by Sancisi (1970; Figure 3). Figure $1 \mathrm{~b}$ shows the distribution of $T_{\mathrm{b}}$ in the latitude-velocity plane at constant galactic longitude, $l=353^{\circ}$, across the area of the association Sco OB2. The Hi feature discussed here is the ridge of high intensity and low velocity dispersion seen between $b=+17^{\circ}$ and $b=+27^{\circ}$ with peak velocity ranging from -1 to $+3 \mathrm{~km} \mathrm{~s}^{-1}$.

The three-dimensional structure of the shell in Perseus can also be seen in Figures $2 \mathrm{a}$ and $\mathrm{b}$, which give the distribution of $\mathrm{H}$ i column density in two different velocity intervals. Map (a) (velocity range from -7 to $0 \mathrm{~km} \mathrm{~s}^{-1}$ ) shows in the direction of Per OB2 an empty region, from which all the gas has been swept away; around it one can see some of the gas which is presumably moving away in directions perpendicular to the line of sight. The large $\mathrm{H}_{\mathrm{I}}$ concentration in the centre of map (b) (velocity range +8 to $\left.+15 \mathrm{~km} \mathrm{~s}^{-1}\right)$ is the receding part of the dense semispherical shell.

The parameters found for these dense shells are given in Table II. The actual masses and densities may be larger, perhaps by a factor of two, as undetected amounts of cool $\mathrm{H}_{\mathrm{I}}$ and molecules may also be present. For example, the $\mathrm{H}_{2}$ molecules observed by Carruthers (1970) in the direction of the star $\xi$ Per may well be associated with the H I shell in Perseus. The values of the densities are probably further underestimated, because the adopted values for the shell thickness may be too large. The clouds of dust seen in these regions are probably associated with the hydrogen shells; in fact position 


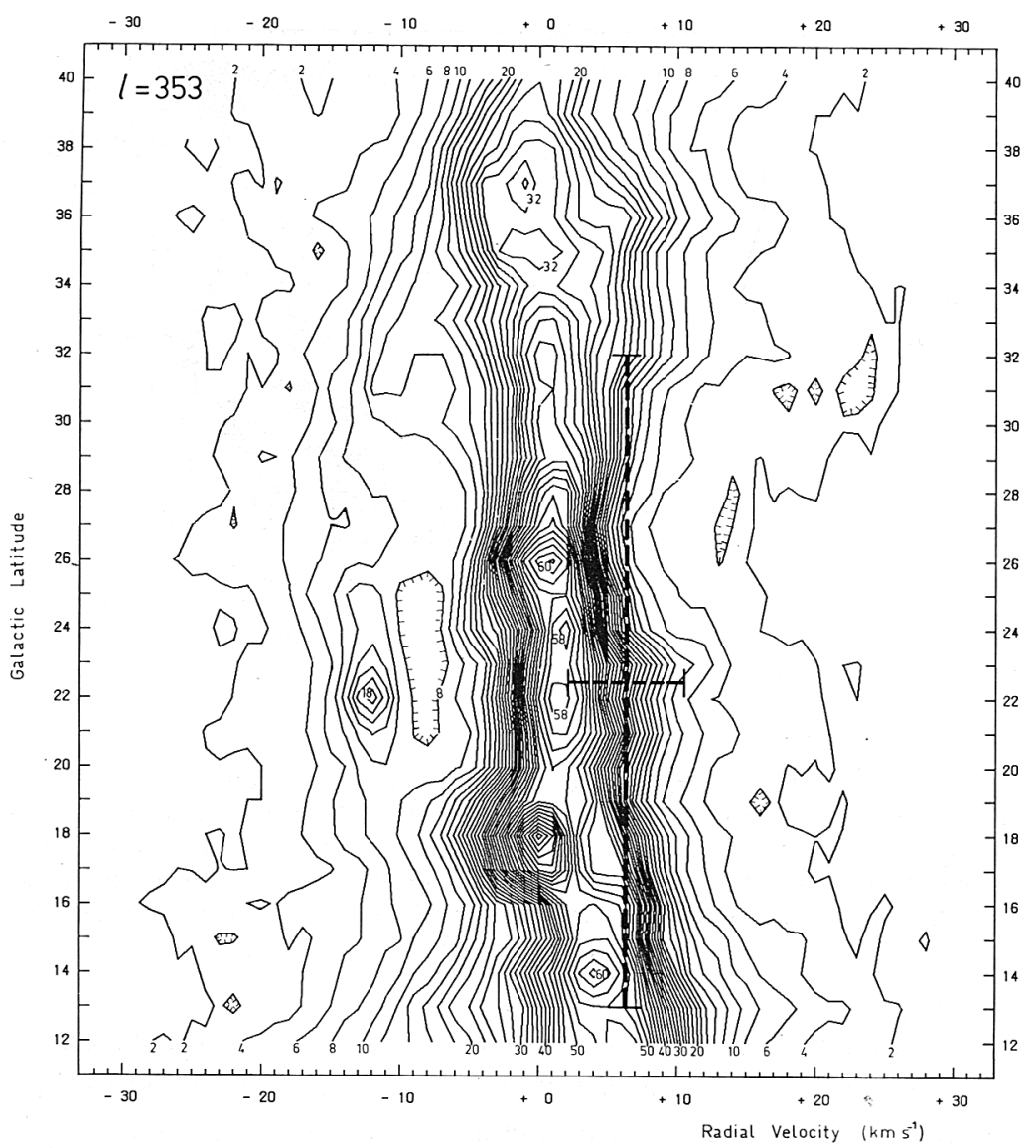

Fig. 1b. Latitude-velocity diagram at constant galactic longitude $\left(l=353^{\circ}\right)$ through the association Sco OB2. The dense shell of semispherical shape discussed in the text is the feature of high intensity and low velocity dispersion seen between $b=+17^{\circ}$ and $b=+27^{\circ}$, with peak velocity from -1 to $+3 \mathrm{~km} \mathrm{~s}^{-1}$. The concentration at $V=-12 \mathrm{~km} \mathrm{~s}^{-1}$ (Sancisi and van Woerden, 1970) is the part of the low-density shell, which moves towards the Sun. The mean radial velocity and latitude extent of Sco OB2 are given by the vertical broken line; the horizontal line gives the average velocity residual.

and extent on the sky of $\mathrm{HI}$ and dust agree closely. In Perseus this agreement is particularly striking, as a comparison of Figures $2 b$ and $c$ shows. In this region the spatial and physical correlation of dust and $\mathrm{H}_{\mathrm{I}}$ is also supported by the excellent agreement between the radial velocity of the $\mathrm{HI}$ shell and that $\left(+8,+9 \mathrm{~km} \mathrm{~s}^{-1}\right)$ of the 'normal' $\mathrm{OH}$ emission, which has been detected recently in the direction of some of these dust concentrations (Crutcher, 1972; Knapp and Kerr, 1973). In Scorpius the correlation of dust and $\mathrm{H} \mathrm{I}$ is particularly well established in the 'Nebula' region (close to $\varrho \mathrm{Oph}$ ), which forms the low latitude edge of the shell. We note also that a number of small concentrations of dust, which are similar to Bok's globules, are present in these regions.

Other, smaller shell pieces have also been tentatively identified as belonging to the same system of gas. Their brightness temperatures are from 4 to $20 \mathrm{~K}$; the masses 

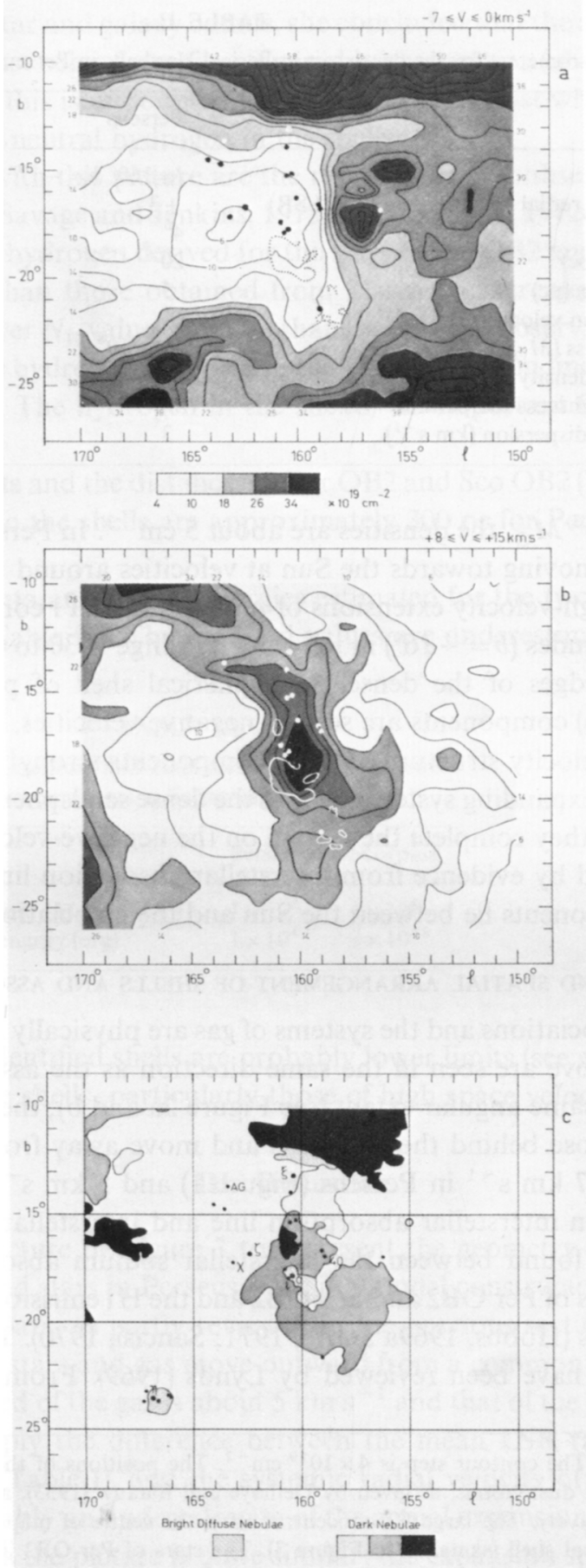

Fig. 2. The maps (a) and (b) show the distribution of the column density of neutral hydrogen in Perseus in two different velocity intervals, respectively -7 to $0 \mathrm{~km} \mathrm{~s}^{-1}$ and +8 to $+15 \mathrm{~km} \mathrm{~s}^{-1}$; the gas was 
TABLE II

Parameters for the dense semispherical Hi shells in Perseus and in Scorpius

\begin{tabular}{lll}
\hline Property & Perseus & Scorpius \\
\hline Centre & $l=160^{\circ} b=-17^{\circ}$ & $l=354^{\circ} b=+23$ \\
Systemic radial velocity $\left(\mathrm{km} \mathrm{s}^{-1}\right)$ (LSR) & +3 & -1 \\
Radius & $4^{\circ}$ & 5 \\
Radius $(\mathrm{pc})$ & 20 & 13 \\
Thickness $(\mathrm{pc})$ & 5 & 5 \\
Expansion velocity $\left(\mathrm{km} \mathrm{s}^{-1}\right)$ & 5 & 3 \\
Total mass $\left(M_{\odot}\right)$ & $1.4 \times 10^{4}$ & $3 \times 10^{3}$ \\
Volume density $\left(\mathrm{cm}^{-3}\right)$ & 30 & 30 \\
Peak brightness temperature $(\mathrm{K})$ & 40 & 40 \\
Velocity dispersion $\left(\mathrm{km} \mathrm{s}^{-1}\right)$ & 3 & 2 \\
\end{tabular}

range from 10 to $10^{3} M_{\odot}$, the densities are about $5 \mathrm{~cm}^{-3}$. In Perseus there are traces of gas (Figure 1a) moving towards the Sun at velocities around $-45,-50 \mathrm{~km} \mathrm{~s}^{-1}$; these seem to be high-velocity extensions of more intense $\mathrm{H}$ I components which are visible at lower latitudes $\left(b=-16^{\circ}\right)$ in the velocity range -30 to $0 \mathrm{~km} \mathrm{~s}^{-1}$; the latter connect with the edges of the denser semispherical shell of positive velocity. In Scorpius (Figure $1 \mathrm{~b}$ ) components are seen at negative velocities, up to $-12 \mathrm{~km} \mathrm{~s}^{-1}$.

The space and velocity structure of these components strongly suggests that they belong to the same expanding system of gas as the dense semispherical shells discussed above; in Scorpius they complete the system on the negative-velocity side. This view is further supported by evidence from interstellar absorption lines, which indicates that these Hi components lie between the Sun and the associations.

\section{(b) KINEMATICAL AND SPATIAL ARRANGEMENT OF SHELLS AND ASSOCIATIONS}

The stars of the associations and the systems of gas are physically related. The densest shells described above are seen in the same direction as the associations and have approximately the same angular extent (see Figure 2a and b); there is good evidence that the stars lie close behind the HI shells and move away from them with mean velocities of about $7 \mathrm{~km} \mathrm{~s}^{-1}$ in Perseus (Figure 1) and $5 \mathrm{~km} \mathrm{~s}^{-1}$ in Scorpius. This evidence is based on interstellar absorption line and interstellar extinction data. A close agreement is found between the interstellar sodium absorption lines in the spectra of some stars of Per OB2 and Sco OB2 and the HI emission components from the expanding shells (Hobbs, 1969a and b, 1971; Sancisi, 1970). The optical data for the Perseus region have been reviewed by Lynds (1969). From analysis of colour

assumed optically thin. The contour step is $4 \times 10^{19} \mathrm{~cm}^{-2}$. The positions of the stars of Per OB2 and an outline of the densest dust clouds, as given by Delhaye and Blaauw (1953), are also marked by dots and dashed lines respectively. The large $\mathrm{HI}$ concentration in the centre of map (b) is the receding part of the dense semispherical shell (sketched in Figure 3). The stars of Per OB2 lie behind this hydrogen and move away from it with a mean recession velocity of about $7 \mathrm{~km} \mathrm{~s}^{-1}$. Map (c) is a reproduction from Khavtassi (1960). The dark nebulosity seen in the centre is probably associated with the $\mathrm{H}_{\mathrm{I}}$ shell: it has approximately the same position and extent as the $\mathrm{HI}$ feature shown in (b). 
excesses as well as star and galaxy counts, she concluded that the association Per OB2 lies adjacent to or just behind a region of variable obscuration (absorption 1 to $3 \mathrm{mag}$ ). On the other hand this is undoubtedly the same layer of dust which was found to be associated with the neutral hydrogen in the shells.

Also consistent with this picture are the results of recent observations of the Ly- $\alpha$ line in absorption (Savage and Jenkins, 1972; Morton et al., 1972a and b); the surface densities of neutral hydrogen derived for the stars of Per OB2 and Sco OB2 are equal to or even larger than those obtained from $21-\mathrm{cm}$ measurements, contrary to the general result of lower $N_{\mathrm{H}}$ values from $\mathrm{L} \alpha$ than from $21-\mathrm{cm}$ observations. This strongly suggests that all the hydrogen observed in the $21-\mathrm{cm}$ emission line lies in front of each of the associations. The hydrogen in the shell is an important fraction of this total column density.

From these results and the distances to Per OB2 and Sco OB2 (Table I) we can infer that the distances to the shells are approximately $300 \mathrm{pc}$ for Perseus and $150 \mathrm{pc}$ for Scorpius.

The total momenta and kinetic energies estimated for the two systems of gas and stars are given in Table III. The reported values are underestimates, however, since

TABLE III

Total momentum and kinetic energy of the systems of gas and stars in Scorpius and Perseus

\begin{tabular}{lll}
\hline & Perseus & Scorpius \\
\hline Momentum $\left(M_{\odot} \mathrm{km} \mathrm{s}^{-1}\right)$ & $1 \times 10^{5}$ & $4 \times 10^{4}$ \\
Kinetic energy (erg) & $1 \times 10^{49}$ & $3 \times 10^{48}$
\end{tabular}

the masses of the identified shells are probably lower limits (see above), and certainly not all pieces of the shells, particularly those of high space velocity, have been identified.

\section{Discussion}

We propose the picture in Figure 3 to represent the geometry and the kinematical behaviour of gas and stars in Perseus. This is a model constructed from the available optical and radio data, as partly reviewed in the previous section; moreover, it has been assumed that stars and gas move outward from a common centre of expansion. The expansion speed of the gas is about $5 \mathrm{~km} \mathrm{~s}^{-1}$ and that of the stars $12 \mathrm{~km} \mathrm{~s}^{-1}$. The latter value is simply the difference between the mean LSR radial velocity of the stars of Per OB2 (Table I), and the systemic radial velocity of the shell (Table II); therefore it is probably an underestimate of the actual expansion velocity of the association. In Scorpius the picture is quite similar; the expansion speeds are 3 and $8 \mathrm{~km}$ $\mathrm{s}^{-1}$ for gas and stars, respectively. Of course this simple semispherical picture represents only a rough approximation of the actual spatial and kinematic configuration 
of stars and gas; in particular, the $\mathrm{H}_{\mathrm{I}}$ shell is quite irregular in shape and morphology.

The picture suggests a few main problems for discussion: (a) the source and input mechanism of kinetic energy, (b) the ages of the shells, and (c) the process of formation of the associations.

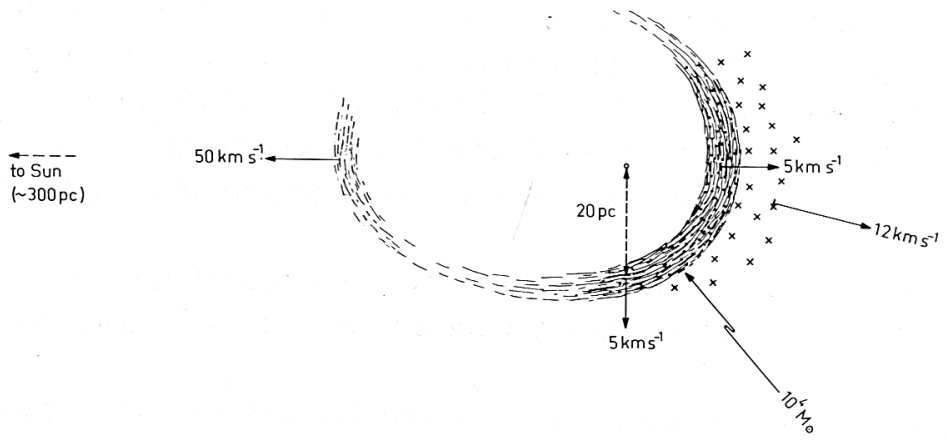

Fig. 3. Sketch representing, in a plane containing the line of sight to the association, the proposed spatial arrangement and state of motion of the gas, dust and stars (X's) in the region of Per OB2. Stars, gas and dust move out from a common centre; the stars have larger velocities than the gas, and move ahead of it.

\section{(a) SOURCE OF KINETIC ENERGY}

Almost twenty years ago it was suggested for the first time that supernova shells (Öpik, 1953) or newly born O stars (Oort, 1954) could account for the observed motion of interstellar gas clouds and for the formation and acceleration of OB associations. But conclusive observational evidence for gas shells related to stellar associations was never found. Gas shells connected with the associations Per OB2 and Sco OB2 have been observed now, but no direct evidence has been found to support either of those two mechanisms. For instance, no radio -continuum or optical emission are found showing any obvious large-scale connection with the expanding shells.*

In the case of the $\mathrm{O}$ star ('old H II region') mechanism various difficulties arise; in particular it is doubtful (Kahn, 1967) whether, in the presence of high densities and efficient cooling, $\mathrm{H}$ II regions can release sufficient amounts of 'organized' kinetic energy. Also, known $\mathrm{H}$ II regions show no clear evidence of a coherent pattern of expanding $\mathrm{H}_{\mathrm{I}}$ motions of the kind observed in the present two cases; in general in the direction of $\mathrm{HII}_{\mathrm{II}}$ regions one observes only an increased internal motion of $\mathrm{HI}$ and a broadening of the $21-\mathrm{cm}$ line profile.

An alternative, more likely interpretation is that the $\mathrm{H}$ I shells observed in Perseus and Scorpius are 'very old' supernova remnants, which have been strongly decelerated by a dense, large interstellar cloud. This hypothesis may account for the observed

* H $\alpha$ photographs shown by G. Monnet (Observatoire de Marseille) during this Symposium indicate that a weak $\mathrm{H} \alpha$ ring is probably associated with the $\mathrm{H}_{\mathrm{I}}$ shell in Scorpius. 
total kinetic energies and radial momenta of the expanding systems (Table III) and also for their shape and morphology.

(b) AGES OF THE SHELLS

For the determination of the ages of the shells from the observed radii and expansion velocities (Table II), it is necessary to make assumptions about the mechanism of expansion of the gas.

If the expansion speed is assumed constant in time, ages of $4 \times 10^{6} \mathrm{yr}$ are derived for the Perseus and Scorpius shells. On the other hand, if the shells are supernova remnants strongly decelerated by interstellar gas, as is most likely in the present case, a 'snow-plow' approximation gives ages of the order of $1 \times 10^{6} \mathrm{yr}$.

It should be realized that these values of the ages are obtained from radii and expansion velocities which are not determined precisely, but are based on the rough approximation of shells having semispherical shape. Therefore every age estimate has an intrinsic uncertainty of perhaps a factor of two.

The estimated ages and the small expansion velocities suggest that these shells may be at the last stage of evolution of a supernova remnant before it loses its identity and merges into the interstellar medium. Therefore any optical, radio or extended $\mathrm{X}$-ray emission from these objects is expected to be totally absent or very weak. Also, none of the pulsars discovered to date lies in the direction of the shells; of course, a pulsar might well have moved quite far from the shell in such a long time since its birth. The only objects in the regions of sky of Per OB2 and Sco OB2 that, as far as we know, might be related with supernova events, are the X-ray sources Sco X-1 and $2 \mathrm{U}$ $0352+30$. Sco $X-1$ is in the direction of one edge of the shell in Scorpius, and $2 U$ $0352+30$ lies in the direction of the centre of the shell in Perseus.

The dimensions of these shells (Table II) are rather small if compared to those of younger supernova remnants, like, for instance, the Cygnus Loop. But the ambient densities of interstellar gas in the present cases are at least 10 to $20 \mathrm{~cm}^{-3}$, i.e., approximately 10 to 100 times larger than the ambient densities found for the Cygnus Loop.

It should be noted that the whole expanding systems of gas described in the first section include also the smaller, higher-velocity shell pieces like the ones at $-12 \mathrm{~km} \mathrm{~s}^{-1}$ in Scorpius and at $-50 \mathrm{~km} \mathrm{~s}^{-1}$ in Perseus; obviously they have larger dimensions than the dense, semispherical shells of Table II.

Finally we note that the supernova outbursts, suggested here to explain the existence of the HI shells, may be the same events which were advocated by Blaauw (1961) in these two regions in order to explain the existence of the runaway stars $\xi$ Per and $\zeta$ Oph. These stars are connected respectively with Per OB2 and Sco OB2. The hypothesis of a common origin of shells and runaway stars is consistent with the kinematical ages of $\xi$ Per and $\zeta$ Oph (1.6 and $1.1 \times 10^{6} \mathrm{yr}$, respectively), which are similar to those of the shells. Alternatively, the supernova outbursts which possibly released these runaway stars may have occurred after the associations were formed; in that case they would have no connection at all with the formation of the observed shells, which in our view must be older than the associations. 
(c) FORMATION OF THE ASSOCIATIONS

The geometry and the motion of stars and gas described above and represented in Figure 3 strongly suggest that the formation of the associations Per OB2 and Sco OB2 is closely related to the expanding, dense shells. The following sequence of events may explain the observations: (1) the supernova remnant sweeps up dense interstellar gas and is decelerated; (2) star formation occurs in the decelerated, condensed shell; (3a) the shell of gas sweeps up more interstellar material and is further slowed down; (3b) the stars move on with constant velocity and therefore are separated from the gas.

From the observational data we can set restrictions on the time scale of star formation. In our view the period of time elapsed since the moment of star formation should be the same as that taken by the gas shell to slow down from the stellar velocities $\left(12 \mathrm{~km} \mathrm{~s}^{-1}\right)$ to the present gas velocity $\left(5 \mathrm{~km} \mathrm{~s}^{-1}\right)$. In the snow-plow approximation, assuming a constant density of the ambient gas, this further deceleration of the shell should have taken about $0.7 \times 10^{6} \mathrm{yr}$ for both Per and Sco. Consequently, the ages of the stars should also be $0.7 \times 10^{6} \mathrm{yr}$. Of course this determination is not very precise; the actual ages of Per OB2 and Sco OB2 may be somewhat larger, but not larger than those derived above for the shells, which are in the range 1 to $4 \times 10^{6} \mathrm{yr}$.

Since estimates of the ages of these associations from kinematical data and from color-magnitude diagrams are available (Table I), a comparison with the present results is interesting. The kinematic ages of the associations should be equal to the ages of the shells; but it must be noted that the former were derived from the simple assumption of constant expansion speed of the stars, while in the model proposed here the present expansion of the association must be regarded as the result of two distinct phases: one of decelerated motion of the shell before the stars are formed, and a second in which the stars move with constant speed equal to that observed now. Therefore, according to this model, the reported values (Table I) of the kinematic ages of Per OB2 and Sco OB2 would be too large and should be reduced by about a factor of 1.5 or 2 before they are compared with the ages of the decelerated shells. These corrections, however, are not very critical for the present comparison. Considering the uncertainties, the agreement between the kinematical ages of the associations and the ages of the shells is quite good, especially for Per OB2. On the other hand, contrary to expectation, the ages derived from the $c-m$ diagrams are larger than the 'time since formation' derived here; they are even larger than the kinematic ages, as already pointed out by Lesh (1969) and discussed by Maeder (1972), and larger than the ages of the shells.

For how long has star formation been going on? So far, we have assumed that all stars have formed and separated from the gas almost at the same time in the stage of the expansion, when the velocity of the shell was around $12 \mathrm{~km} \mathrm{~s}^{-1}$ in Perseus and $8 \mathrm{~km} \mathrm{~s}^{-1}$ in Scorpius. The large spread of stellar velocities suggests that the actual situation is more complicated. In fact, the total spread of the line-of-sight velocities of the stars, from about +6 to $+19 \mathrm{~km} \mathrm{~s}^{-1}$ in Per and from -1 to $+19 \mathrm{~km} \mathrm{~s}^{-1}$ in Sco (with respect to the centre of expansion), does not seem to be entirely accounted 
or by intrinsic errors, random motions and expansion, particularly in the case of the issociation Per OB2. It is possible, therefore, that this large velocity spread is partly :he result of a formation process extending in time, and perhaps still going on. This would imply a corresponding spread in the ages of the stars and a correlation between he ages and the space velocities of the stars. In this connection we point out also that ihe runaway star $\xi$ Per which is moving in the same direction as the other association stars with a line of sight velocity of $+64 \mathrm{~km} \mathrm{~s}^{-1}$, could represent a case of star formation at an earlier stage of expansion of the shell, when the latter still had a high velocity 'about $64 \mathrm{~km} \mathrm{~s}^{-1}$ ). This would obviously conflict with the 'proto-binary' hypothesis 'Blaauw, 1961), which is the generally accepted explanation for the 'runaway' phenomenon.

We further note that groups of T-Tauri stars are found in the regions of Per OB2 'close to IC348, Herbig, 1954) and Sco OB2 (Herbig, 1962). They are associated with dust concentrations, which are probably local condensations of the expanding shells; if they had formed before the process of expansion started, presumably their parent interstellar material would have been blown away by the event which caused the expansion. Therefore, it is likely that these stars have been forming quite recently, after the OB stars, in the dense and cool regions of the shells. Unfortunately, proper motions and radial velocities of these low-luminosity stars are not available, and their possible connection with the shell cannot be tested.

Finally we note that the braking inferred from the velocity decrease of the gas shells with respect to the stars, from 12 to $5 \mathrm{~km} \mathrm{~s}^{-1}$ in Perseus and from 8 to $3 \mathrm{~km} \mathrm{~s}^{-1}$ in Scorpius, implies densities, 10 to $20 \mathrm{~cm}^{-3}$, which are quite normal for interstellar clouds. The present mean separation in space between stars and shell, resulting from the deceleration of the latter, is inferred to be only a few parsec. Such a closeness in space of stars and shells is consistent with the presence in the expanding shells of $\mathrm{H}_{\mathrm{II}}$ regions excited by the stars of the associations, such as $\delta$ Sco and $\pi$ Sco (Sharpless, 1959), and of bright reflection nebulosity (around o Per).

\section{Concluding Remarks}

The results of this work bring some new elements for a discussion of : (a) the kinematics of Per OB2 and Sco OB2, (b) the connection of associations with interstellar matter, and (c) the expansion of the Gould Belt system.

(a) The geometry and the motion of the shell at the time of star formation account for: (i) the presence and the rate of expansion of Per OB2 and Sco OB2, and (ii) their mean recession velocities, which are, as pointed out by Blaauw (1970), anomalously high compared with the general interstellar medium and the average B-star population in the solar neighborhood. In our view these recession velocities represent the component of the expansion of the associations in the direction of the line of sight, away from the Sun (Figure 3). Star formation occurred only in the densest shell of the expanding system, the one receding from the Sun; therefore the resulting picture is largely different from the conventional one of an approximately spherical system of stars expanding in all directions. 
(b) Per OB2 and Sco OB2 probably are not the only associations having such a physical connection with interstellar matter. In fact, similar expanding shells are also found in the associations Lac OB1 (part of our 21-cm survey in associations) and Cep OB3 (van Someren Greve, private communication), but the nature of their connection with the stars is more difficult to establish. In some cases the identification of such shells may be very difficult. For instance, successive events of the type discussed above, which are quite likely to occur in the lifetime of an association, would produce a very chaotic situation in the interstellar medium; this may have happened in the Orion region, where star formation has been going on for a long time, and stars of quite different ages are present. On the other hand, in such successive events the history of Per OB2 and Sco OB2 may well repeat itself and lead to the formation of subgroups of different ages and to the shifting of the place of star formation, which were discussed by Blaauw $(1962,1964)$.

(c) The recognition of the peculiar origin and nature of the large space motions of Per OB2 and Sco OB2 also has some direct consequence on studies of the kinematics of the Gould Belt system. The inclusion (see for instance Lesh, 1968) of these two - and possibly also of the other - associations, together with all Gould Belt stars, in solutions for the general expansion of the system may not be physically justified and may seriously affect the results.

To conclude we point out that probably these shells contain fairly large amounts of molecules; detailed surveys of $\mathrm{OH}, \mathrm{H}_{2} \mathrm{CO}$ and $\mathrm{CO}$ in these regions would be of great interest and may help unravel the kinematic structure of the shells.

\section{References}

Blaauw, A.: 1960, Nuffic Intern. Summer Course on Present Problems Concerning the Structure and Evolution of the Galactic System (The Hague, The Netherlands).

Blaauw, A.: 1961, Bull. Astron. Inst. Neth. 15, 265.

Blaauw, A.: 1962, in L. Woltjer (ed.), Interstellar Matter in Galaxies, W. A. Benjamin, Inc., New York. p. 32.

Blaauw, A.: 1964, Ann. Rev. Astron. Astrophys. 2, 213.

Blaauw, A.: 1970, in W. Becker and G. Contopoulos (eds.), 'The Spiral Structure of our Galaxy', IAU Symp. 38, 199.

Carruthers, G. R.: 1970, Astrophys. J. Letters 161, L81.

Crutcher, R. M.: 1972, Ph.D. Thesis, University of California, Los Angeles.

Delhaye, J. and Blaauw, A.: 1953, Bull. Astron. Inst. Neth. 12, 72.

Herbig, G. H.: 1954, Publ. Astron. Soc. Pacific 66, 19.

Herbig, G. H.: 1962, Adv. Astron. Astrophys. 1, 47.

Hobbs, L. M.: 1969a, Astrophys. J. 157, 135.

Hobbs, L. M.: 1969b, Astrophys. J. 158, 461.

Hobbs, L. M.: 1971, Astrophys. J. 166, 333.

Kahn, F. D.: 1967, in H. van Woerden (ed.), 'Radio Astronomy and the Galactic System', IAU Symp. 31, paper 15.

Khavtassi, J.: 1960, Atlas of Galactic Nebulae, Abastumani Astrophysical Observatory.

Knapp, G. R. and Kerr, F. J.: 1973, Astron. J. 78, 453.

Lesh, J. R.: 1968, Astrophys. J. Suppl. 16, 371.

Lesh, J. R.: 1969, Astron. J. 74, 891.

Lynds, B. T. : 1969, Publ. Astron. Soc. Pacific 81, 496. 
Maeder, A.: 1972, in G. Cayrel de Strobel and A. M. Delplace (eds.), 'Age des Étoiles', IAU Colloq. 17 XXIV-1, Observatoire de Paris-Meudon.

Morton, D. C., Jenkins, E. B., Matilsky, T. A., and York, D. G.: 1972a, Astrophys. J. 177, 219.

Morton, D. C., Jenkins, E. B., and Macy, W. W.: 1972b, Astrophys. J. 177, 235.

Oort, J. H.: 1954, Bull. Astron. Inst. Neth. 12, 177.

Öpik, E. J.: 1953, Irish Astron. J. 2, 219.

Raimond, E.: 1966, Bull. Astron. Inst. Neth. 18, 191.

Sancisi, R.: 1970, Astron. Astrophys. 4, 387.

Sancisi, R. and van Woerden, H.: 1970, Astron. Astrophys. 5, 135.

Savage, B. D. and Jenkins, E. B.: 1972, Astrophys. J. 172, 491.

Sharpless, S.: 1959, Astrophys. J. Suppl. 4, 257.

\section{R. Sancisi}

\section{Kapteyn Astronomical Institute,} University of Groningen, Groningen, The Netherlands

\section{DISCUSSION}

$J$. R. Dickel: A younger example of such an expanding $21-\mathrm{cm}$ cloud is that around the northeast part of the SNR HB21. (Assousa and Erkes, Astron. J., in press). It is also of interest that the position of the densest part of the $\mathrm{HI}_{\mathrm{I}}$ cloud is also the region of HB21 where there is an apparent turnover in the lowfrequency spectrum of the source. If the latter is interpreted as free-free absorption and if the ionized and neutral regions are coincident the gas must be cold and about $10 \%$ ionized at the current time.

Hughes: There appears to be further evidence for expanding rings of $\mathrm{H} I$ with associated star formation. Rickard in 1968 detected one in the Cas-Per arm from $21-\mathrm{cm}$ observations and showed that the motion of early-type stars agreed with the HI velocities. Some of our more recent work (V. A. Hughes and D. Routledge, 1972, Astron. J. 77, (1972), 210) showed evidence for a similar expanding ring of HI surrounding the Sun, which also appeared to form an envelope for the dust in the solar neighborhood and which may also be associated with Gould's belt.

Monnet: There is considerable evidence for shells in external galaxies - when there are early-type stars to ionize them. They have been observed in $\mathrm{H} \alpha$ by Baade in NGC 6822 and K 342, and I have found six of them in M33. A curious point is that they are observed only in the borders of the galaxy - as well as the shells you have observed in Perseus.

Sancisi: Expanding shells of neutral hydrogen have been reported several times in the past, with or without any connection with stars and stellar associations. However, I want to emphasize that in order to establish the reality of expanding shells a careful study of the spatial and velocity structure of the neutral hydrogen is necessary. In the present case also the physical relationship between the expanding shell and the stellar association has been studied.

Greenberg: I would like to suggest that this shell region you have begun to study would be an interesting place to study the way in which the dust appears or is modified in a condensation. The time scale you mentioned $\left(\sim 2 \times 10^{6} \mathrm{yr}\right)$ would be sufficient to have permitted a very substantial accretion onto grains. It would therefore appear useful to examine this specific region for the wavelength dependence of the extinction to see if it is modified from 'normal' or not. What is the hydrogen density there?

Sancisi: This would be normal, because the dust has been gathered up so that we get an increase in surface density of the dust particles. With column densities $\sim 5 \times 10^{20} \mathrm{~cm}^{-2}$ and a thickness $\sim 5 \mathrm{pc}$ the density is only $\sim 30 \mathrm{~cm}^{-3}$. This probably is a low value because it has been obtained assuming the gas to be optically thin, and probably the thickness of the shell is larger.

Jenkins: Ly- $\alpha$ absorption data taken by OAO-2 for stars in the Persei OB2 aggregate indicate $\mathrm{HI}$ column densities generally in excess of $10^{21} \mathrm{~cm}^{-2}$. These results suggest that significant saturation is probably occurring with the $21-\mathrm{cm}$ radiation since your maximum column densities seem to be around $5 \times 10^{20} \mathrm{~cm}^{-2}$.

Sancisi: The column density of $5 \times 10^{20} \mathrm{~cm}^{-2}$ refers only to the $\mathrm{H}$ I shell. The total $\mathrm{HI}$ column density from $21-\mathrm{cm}$ observations in the direction of the Per OB2 stars is about the same as derived from $L y-\alpha$ absorption. This probably means that all the $\mathrm{H}_{\mathrm{I}}$ gas in that direction is located between the stars of Per OB2 
and the Sun. Some saturation may certainly be occurring with the $21-\mathrm{cm}$ radiation from the shell, but our 21-cm data do not give any clear indication in that sense.

Mills: Can you give any information about the energetics of the events which produce these expanding shells?

Sancisi: The only relevant information I have is the observed present total kinetic energy of the expanding system, about $1 \times 10^{49} \mathrm{erg}$, and the total radial momentum of the order of $10^{5} M_{\odot} \mathrm{km} \mathrm{s}^{-1}$. It is likely, however, that these values are underestimates. They seem to be of the same order as expected for very old $\left(\sim 1 \times 10^{6} \mathrm{yr}\right)$ type II supernova remnants. 